


\title{
NOTE E RASSEGNE
}

\section{UN IOMO DI CARATTERE DI PAOLA CAPRIOLO:}

\author{
IL MOVIMENTO IMPOSSIBILE
}

di

Giovanna De Luca

Nei romanzi di Paola Capriolo i pensieri e le azioni dei suoi personaggi convergono verso un'idea principale: l'impossibilità dell'essere umano di poter esperire la realtà. Questa incapacità viene espressa attraverso l'uso di una scrittura asfittica, necrofila, unico elemento della realtà di cui si è capaci di fare esperienza. Essa è autoreferenziale. narcisistica: una scrittura allo specchio, dove lo scrivere diventa il soggetto della scrittura. L'estetizzazione della lingua, la sua stilizzazione si trasformano in un "mito stilistico" che diventa l'unico credo a cui l'uono riesce ad aggrapparsi. Usando la chiave interpretativa nietzschiana, suggerita implicitamente dalla stessa autrice nel frontespizio del romanzo $\mathrm{L}$ womo di carattere, possiamo dire allora che la Capriolo attribuisce alla scrittura, in quanto arte, il valore di "giustificazione estetica dell'esistenza." (Come dice la prefazione del 1886 alla riedizione della Nascita della tragedia di Nietzsche.)

"C'è un solo mondo, ed è falso, crudele, contraddittorio, corruttore, senza senso [...]. Un mondo così fatto è il vero mondo [...]. Noi abbiamo bisogno della menzogna per vincere questa "verità", cioè per vivere [...]. La metafisica, la morale, la religione, la scienza - vengono prese in considerazione solo come diverse forme di menzogna: col loro sussidio si crede nella vita. "la vita deve ispirare fiducia": il compito, cosí posto, è immenso. Per assolverlo l'uomo dev'essere per natura un mentitore, deve essere prima di ogni altra cosa un artista. (Nietzsche 115)

I personaggi dei romanzi della Capriolo non credono nello sviluppo e nel futuro, la loro sfera d'azione voriebbe essere in un tempo ideale, ma ciò che effettivamente vivono è invece una intollerabile presente realtà. È quest eterna ambiguità che caratterizza le loro esistenze e di conseguenzá il loro destino si risolve in una schizofrenica staticita. Usando ancora una volta come punto di riferimento Nietzsche si può affermare che questa loro incapacità è determinata dalla consapevolezza dell' "eterno ritorno dell'eguale" orvero la negazione della linearità del tempo, della sua composizione di presente, pas- 
sato e futuro secondo la visione "storica" stabilita dalla tradizione giudaicocristiana. La dottrina di Nietzsche a questo atteggiamento nichilistico dà una soluzione abbastanza ottimista (Cosi parlò Zaratbustra) in quanto riconosce all'applicazione della volontà di potenza posseduta da ogni singolo uomo, la facoltà di prendere delle decisioni e di far sì che ogni momento del tempo reale abbia tutto il suo senso in sè. I personaggi della Capriolo riescono a dare un senso solo a ciò che è al di là di essi, al metafisico, esercitando così la volontà di potenza in una sfera ideale invece che reale. "La conoscenza, il dire sì alla realtà, è per il forte una necessità pari a quella che è per il debole, per ispirazione della propria debolezza, la vigliaccheria e la fuga davanti alla realtà - l' 'ideale" (Vattimo 38).

La scrittura della Capriolo è spesso rivolta al passato, vagamente definito, che meglio si presta a suscitare emozioni in un mondo di morte. Parafrasando il concetto d'arte di Nietzsche in Umano troppo umano, Gianni Vattimo scrive: "[L']arte per agire sugli animi ha bisogno di un mondo che che non è più il nostro; se vuole mantenersi nel nostro mondo, deve richiamarsi al passato, ricreare artificialmente oggi le condizioni che la rendevano attuale in altre epoche" (41).

Per la scrittrice, il passato, il presente e il futuro sono morte, meta e destino di personaggi il cui senso di impotenza nei confronti del reale li incornicia in uno scenario funereo. Ma la morte è allo stesso tempo potenziale ed effettiva realtà alla quale si riesce a dare un significato in quanto rappresenta un cambiamento di status, un movimento. L'autrice così descrive Vulpius, il protagonista del romanzo La spettatrice, sul letto di morte della compagna Dora:

"[S]i sentiva confermato nell'idea che la quiete fosse superiore al movimento, la morte alla vita. Così il macabro spettacolo dal quale gli altri distoglievano gli occhi con orrore costituiva per lui una trasfigurazione miracolosa prima di svanire nel nulla, Dora ascendeva a quell'ineffabile purezza che non è degli esseri umani, ma soltanto dei cristalli e delle statue, di quanto non ha mai posseduto la vita o da essa si è separato." (134)

La morte quindi è realizzazione di quell'ideale estetico impossibile da raggiungere in vita. È l'asfittica armonia raggiunta "nell'altro regno" dalla cliente dell'albergo nel romanzo Il doppio regno, o dall'ingegner Stiler nel giardino senza vita in Un uomo di carattere. I protagonisti dei romanzi non diventano, non si evolvono, sono emotivamente anziani, prossimi alla morte. La scrittura anche è della vecchiaia, dove la consapevolezza d'impotenza si unisce ad una repressione dell'eros, della vita, che ha come conseguenza il thanatos. 
"Larte esplica in via secondaria il compito di conservare e benanche di ricolorite un pò concezioni spente, shiadite; essa allaccial, quando assolve questo compito, un legame intorno a epoche diverse e ne lat ritornare gli spiriti. Per lat veritit e solo unat vita di larval come sopra delle tombe, quella che in tal modo sorge, oppure come il ritorno in sogno di cari morti; ma almeno per alcuni istanti l'antico sentimento si riclesta e il cuore pulsa con un ritmo ormai dimenticato." (Nietzsche 122)

Nel romanzo La spettatrice (Bompiani 1995), l'attore di teatro Vulpius conduce la suá compagna -collega Dora alla morte nel tentativo cli traslormarla nella misteriosa spettatrice che si presenta ogni sera al teatro; Walter nel Nocchiero (Bompiani 1989) sarà portato all morte dal dubbio mai chiarito di avere o no sposato la donna che aveva tanto desiderato; la stabile ospite d'albergo de $I l$ doppio regno (Bompiani 1991), preferisce la permanenza nell'asettica struttura dall'irreale architettura e personale, piuttosto che un ritorno al mondo reale dal quale era venuta; e infine l'ingegnere Stiler in Un uomo di carattere (Bompiani 1996) sceglie di dedicare la sua vita alla creazione di un giardino perfetto ma senza vita, piuttosto che agli affetti personali.

Pur preferendo descizioni ambigue di spazi irreali, (mancano difatti definizioni precise di luoghi,) gli ambienti chiusi sono descritti clall'autrice in maniera dettagliata. Essi diventano strumenti cli separazione tra il singolo individuo ed il resto del mondo. Sono allo stesso tempo rifugio e prigione di personaggi che non sanno come aprirsi alla vita. Abbondano descrizioni di grancli alberghi, come nel Noccbiero e il Doppio Regno, nobili palazzi antichi come in Vissi damore (Bompiani 1992) e La grande Enlalia (Feltrinelli 1988): teatri di provincia e teatri d'opera presenti rispettivamente ne La spettatrice e Vissi d'amore o il giardino come nell'ultimo romanzo Ĺn tomo di carattere, che sebbene sia all'aperto, non ha vista, perchè circondato da un muro e non ha vita perchè gli animali non vi hanno accesso.

Queste descrizioni di ambiente sono accompagnate da scelte di personaggi adeguati appartenenti a ceti sociali e culturali elevati o che ambiscono a tale status come il giovane Walter nel Nocchiero. Sembra che la scrittrice attribuisca un'inabilità di vivere a personaggi raffinati o colti, in contrasto con personaggi di ceto piu basso (il giardiniere e sua moglic Ursula in $\mathrm{C}^{\mathrm{n}}$ Uomo di carattere) che riescono invece a condurre la propria vita regolarmente proprio perchè il loro status non favorisce quel processo di "idealizzazione" del peronaggio erudito o artista.

Al personaggio consapevole clella propria impotenza non rimane altro che incontrarsi con il suo destino. L'autrice riesce così a superare l’inconciliabil- 
ità tra personaggio e fato tipica della narrativa moderna riallacciandosi in chiave contemporanea ai topoi del romanzo ottocentesco, dove il lettore sin dalle prime pagine poteva intuire l'inevitabile conclusione verso la quale andavano incontro i personaggi con le loro azioni e sentimenti. ${ }^{1}$

La Capriolo supera l'incongruenza tra personaggi e destino di cui parla il Debenedetti, accorciando le clistanze esistenti tra essi. Essi non hanno bisogno di compiere determinate azioni per meritare uno specifico fato. Tutti hanno un comune destino: la morte. La loro stessa vita è esperienza di morte, e il sopragiungere clella morte fisica non è altro che il compimento di un finale movimento nel "divenire" che è loro concesso di effettuare.

Nell'ultimo romanzo della Capriolo, Un nomo di carattere, riappare quel gusto decadente e colto presente sin dai racconti della Grande Eulalia, la sua opera prima. La narrativa in prima persona è quella di un giovane romantico pittore, Bausa, il quale durante uno dei suoi annuali soggiorni estivi in un paese di campagna, conosce l'ingegner Erasmo Stiler. L'elemento che avvicina i due personaggi è un incolto giardino, ereditato dall'ingegnere da suo zio, (morto a causa di un ossicino di pollo conficcatosi di traverso nella gola) e visitato spesso dal giovane Bausa attratto dal caotico fascino che il giardino suscita in lui. Stiler invece è attratto dall'idea di poter domare l'incolta e insubordinata vegetazione che cresce nella sua eredità. Bausa da attento spettatore narra la storia della sua amicizia con l'ingegnere e della interminabile lotta intrapresa da quest'ultimo per clare ordine al suo giardino. Il suo obiettivo è quello di portarlo ad una perfezione icteale. Ciò comporta una lotta incessante contro la realtà clalla quale alla fine verrà sopraffatto.

È evidente nello stile letterario della Capriolo un certo scolasticismo. I personaggi sono descritti schematicamente e in maniera molto esplicita senza lasciare spazio all'intuizione del lettore. La struttura narrativa di ln uomo di carattere, ritrae in posizioni antitetiche i due personaggi principali: Bausa è il romantico pittore, pieno di indecisioni sul suo futuro e sulla sua vita. È l'osservatore passivo della realtà che lo circoncla e sopratttutto del dinamico ingegner Stiler che preferisce donchisciottescamente intraprendere un'estenuante lotta contro l'indomabille natura, fino alla fine dei suoi giorni, piuttosto che sottostare alla realtà. Il primo è giovane, il secondo è anziano; l'uno fatalista, l'altro ha invece un atteggiamento pragmatico. essi sono rappresentanti di due mondi, quello dionisiaco e quello apollineo mai complementari tra di loro.

Stiler e Bausa sono dei prototipi, dei personaggi senz'anima (l'autrice volutamente non si sofferma su descrizioni che forniscano un'idea della psicolo- 
gia dei personaggi: ognuno di essi è privato della propria individualita per immergersi in un signilicato cosmico soprattutto con la morte che lo alssorbirà nel tutto) clue aggrappandosi l'uno alla propria volontì, l'altro alla propria rassegnazione cercano invano di dare un senso a ciò che li circonda. Lo stesso si può dire degli altri personaggi delle opere della Capriolo: Vulpius con la forza della propria volontà cerca di trasformare il suo recitare quoticliano in un piccolo teatro di provincia in una recitá ideale dove čè posto solo per la perfezione, mentre Dora si contenta di vivere il presente senza pretese e di amare con venerazione il collega -amante fino allá monte; l'anonima cliente d'albergo ne $/ l$ doppio regno dopo i primi atti di ribellione nel vano tentativo di comprendere il perchè si trovasse li e la logica dietro le azioni degli orwelliani abitanti della sterile nuova dimora (camerieri e direttore), si rassegna allo stile di vita dellalbergo, rinuncia ai "perchè" diventati ormai retaggi scomodi del regno di provenienza. Quando i temporanei clienti dellalbergo, rappresentanti del mondo esterno pieni di certezze e di vita cosi diversi da ciò che è diventata lei, la esortano ad uscire cla quella sorta di prigione, la donna rifiuta fermamente scegliendo di non tornare più indietro.

La narcisistica scrittura autoreferenziale della Capriolo si riflette in $U_{n}$ uomo di carattere nell'artistica ricreazione del giardino da parte dell'ingegner Stiler. La creazione del giardino assume per Stiler lo stesso valore che la scrittura ha per la Capriolo: essa rappresenta, in quanto atto artistico, l'unica arma a disposizione dell'uomo per "non perire a causa della verità". La struttura fisica stessa del giardino rispecchia una visione narcisistica del mondo. Il giardino non ha vista, non ha aperture verso l'esterno. Sebbene sia sentito da Stiler come conquista di uno spazio e come una sfida continua sul mondo naturale, a causa della sua struttura chiusa verso il mondo, non rappresenta (come invece avveniva per il giardino rinascimentale) un elemento di grandezza politica o intellettuale. Data la suá microcosmicità, esso è per l'ingegnere il sogno dell'adamitica sovranità sul mondo. Il giardino diventa un'estensione dell lo del persoanggio (Stiler) e la direzione della libido viene spostata clalla cugina Zelda per riversarsi su se stesso e sul giardino.

L'assenza dal giardino di fontane (quindi del fluire dell'acqua) ce quella di uccelli o altri tipi di creature che popolano generalmente i giardini, gli conferisce staticità. Stiler, nega il fluire del tempo, l'applicazione della sua volontà sul giardino ha come impossibile obiettivo quello di dare un senso finito a ogni attimo della vita, ma le continue battaglie contro la natura, hanno come esito la morte, unico vero senso clella vita. 
"Il suo cosmo era un'artificiosa eccezione ritagliata nel caos, e quest'ultimo lo assediava da ogni parte nello stesso momento in cui le colline assediavano la geometria del giardino con la loro indomita vegetazione. Appunto in ciò stava il fascino dell'impresa di Stiler, e insieme la sua debolezza: sentivo pena per lui, rammentando quanto avesse sacrificato alla propria paura sebbene fosse consapevole che il nulla prima o poi avrebbe finito col prevalere." (Un uomo di carattere 188)

Bausa è lo spettatore dell'opera di Stiler, è il debole che cerca di dare una spiegazione alla realtà. "L'arte non nasconde con le sue forme una qualche 'verità' obiettiva delle cose; essa, invece, come attività di creazione di menzogna si contrappone alla passività, reattività, spirito di vendetta che caratterizza la ricerca della verità" (Vattimo 104).

Il voyerismo di Bausa, come l'attivismo di Stiler, approdano allo stesso risultato. Entrambe portano alla rinuncia di vivere il reale, la vita. Bausa è cosciente fin dall'inizio della sua debolezza, ma non fa niente per cambiare. La sua vita, come la sua pittura e il suo raccontare la storia di Erasmo Stiler fanno parte di una personalità parassita, che consciamente preferisce il non esporsi ai colpi clella realtà conducendo un'esistenza fatte da altrui esistenze. "Terminati gli studi universitari avevo scelto la prefessione dell'insegnante, senza che tale scelta fosse suggerita da una vocazione precisa....[D]el resto quella prefessione, almeno come io la intendevo, sembrava fatta per me, poichè consisteva nel preparare altri a qualcosa che mi guardavo bene dall'affrontare" ( $T$ n ıomo di carattere 188).

Le esistenze di Bausa e di Stiler esprimono una comune incapacità di esperire il reale risultante in una volontà di vivere come osservatore, nel caso di Bausa, e in un aggressivo e continuo attacco al reale nel caso di Stiler. In entrambe I casi I peronaggi assumono posizioni estreme, che sfociano in un solo possibile destino: l'immobilità della morte in vita.

\section{NOTE}

1 Debenedetti nel suo saggio Personaggi e destino, (1959) attribuisce la frammentarietà e conseguente assurdità del personaggio del romanzo moderno, (completamente slegato da qualsiasi schema di azioni risolte in determinati destini) alla consapevolezza dell'uomo di essere rimasto orfano di padre, orvero di non avere più modelli a cui ispirarsi. "Assumendosi la responsabilità di averci messi al mondo, il padre ci rassicurava che vivere era un bene; che i nostri destini favorevoli o arversi, sempre arrebbero finito col modellare le loro sagome sulle linee di forza della vita." La colpevolizzazione del padre era riuscita ad esorcizzare questá mancanza. Terminata però l'epoca della psicoanalisi, con il sopraggiungere dell'esistenzailismo, la colpa è stata scaricata sull'esistenza stessa. La let- 
teratura ha enlatizzato l'assurditì del vivere (Pirandello, Beckett, Camus), e non trovando giustificazioni allesistenza, ha creato un distacco tra personaggi, azioni e loro destino.

\section{OPERE CITATE}

Capriolo, Paola. Un nomo di carattere. Milano: Bompiani, 1996.

La spettatrice. Milano: Bompiani, 1995.

- Vissi d'amore. Milano: Bompiani, 1992.

- Il cloppio regno. Milano: Bompiani,1991.

- Il nocchiero. Milano: Feltrinelli, 1989.

- Grande Eulalia. Milano: Feltrinelli, 1988.

Chessick, Richard. Psychology of the Self and Treatment of Varcissism. New Jersey: Jason Aronson, 1985.

Comito, Terry. The Idea of the Garden in the Reinassance. New Brunswick: Rutgers UP, 1978.

Danto, Arthur. Nietzsche as Philosopher: New York: MacMillan, 1965.

Debenedetti, Giacomo. Personaggi e destino. Milano: Il Saggiatore, 1977.

Jones, Ernest. The Life and the Work of Sigmund Freud. Volume II. New York: Basic Books, 1955.

La Porta, Filippo. La nuova narrativa italiana. Torino: Bollati Boringhieri, 1995.

Memmi, Germaine. Frend et la creation litteraire. Paris: L'Harmattan, 1996.

Nietzsche, Friederich. Umano troppo umano. Ed. Colli, Montinari. Milano: Adelphi, 1967.

Picard, Michel. La lettérature et la morte. Paris: PU F, 1995.

Rowe, Dorothy. The Constmuction of Life and Death. New York: John Wiley \& Sons, 1982.

Vattimo, Gianni. Introduzione a Nietzscbe. Bari: Laterza, 1990. 